\title{
Superoxide Dismutase 3 Limits Collagen-Induced Arthritis in the Absence of Phagocyte Oxidative Burst
}

\author{
Tiina Kelkka, ${ }^{1,2,3}$ Juha Petteri Laurila, ${ }^{1,2}$ Outi Sareila, ${ }^{1}$ Peter Olofsson, ${ }^{4}$ \\ Mikko Olavi Laukkanen, ${ }^{1,5}$ and Rikard Holmdahl, 3 \\ ${ }^{1}$ Medicity Research Laboratory, University of Turku, Tykistökatu 6 A, 20520 Turku, Finland \\ ${ }^{2}$ Turku Doctoral Programme of Biomedical Sciences, Kiinamyllynkatu 13, 20520 Turku, Finland \\ ${ }^{3}$ Medical Inflammation Research, Department of Medical Biochemistry and Biophysics, Karolinska Institutet, \\ 17177 Stockholm, Sweden \\ ${ }^{4}$ Redoxis AB, Sahlgrenska Science Park, Medicinaregatan 8A, 41346 Göteborg, Sweden \\ ${ }^{5}$ Fondazione SDN, 80143 Naples, Italy \\ Correspondence should be addressed to Rikard Holmdahl, rikard.holmdahl@ki.se
}

Received 14 September 2011; Revised 21 November 2011; Accepted 12 December 2011

Academic Editor: Christoph Aufricht

Copyright ( $) 2012$ Tiina Kelkka et al. This is an open access article distributed under the Creative Commons Attribution License, which permits unrestricted use, distribution, and reproduction in any medium, provided the original work is properly cited.

\begin{abstract}
Extracellular superoxide dismutase (SOD3), an enzyme mediating dismutation of superoxide into hydrogen peroxide, has been shown to reduce inflammation by inhibiting macrophage migration into injured tissues. In inflamed tissues, superoxide is produced by the phagocytic NOX2 complex, which consists of the catalytic subunit NOX2 and several regulatory subunits (e.g., NCF1). To analyze whether SOD3 can regulate inflammation in the absence of functional NOX2 complex, we injected an adenoviral vector overexpressing SOD3 directly into the arthritic paws of $N c f 1^{* / *}$ mice with collagen-induced arthritis. SOD3 reduced arthritis severity in both oxidative burst-deficient $N c f 1^{* / *}$ mice and also in wild-type mice. The NOX2 complex independent anti-inflammatory effect of SOD3 was further characterized in peritonitis, and SOD3 was found to reduce macrophage infiltration independently of NOX2 complex functionality. We conclude that the SOD3-mediated anti-inflammatory effect on arthritis and peritonitis operates independently of NOX2 complex derived oxidative burst.
\end{abstract}

\section{Introduction}

Extracellular superoxide dismutase (SOD3) is an enzyme known to catalyze dismutation of the highly reactive, superoxide anion into longer-lived and more stable hydrogen peroxide [1]. The consequences of SOD3 action in the cells reach beyond the antioxidative functions, as it has been shown to downregulate inflammation [2], stimulate cell proliferation during tissue injury recovery [3], and to counteract apoptosis $[4,5]$ by affecting cytokine production, cell signal transduction, and expression of survival-related genes.

The anti-inflammatory properties of SOD3 have been studied in models of pulmonary disease and peritonitis $[2,6]$. Previous work with collagen-induced arthritis (CIA) suggests that both genetic transfer of the SOD3 gene [7] as well as a small molecular SOD mimetic have the ability to ameliorate arthritis [8]. The arthritis ameliorating effect of
SOD3 was later confirmed using SOD3 knock-out mice [9]. These results were explained on the basis that SOD3 acts as an antioxidant and catalyses dismutation of superoxide into hydrogen peroxide, thus reducing inflammation-induced oxidative stress and restoring the oxidant balance in the arthritic joints $[10,11]$. This explanation, however, is difficult to reconcile with the finding that animals naturally deficient in the induced oxidative burst in fact develop more severe arthritis $[12,13]$.

The most potent producer of superoxide, the substrate for SOD3, is the well-characterized phagocytic NOX2 complex. In inflamed tissues NOX2 complex produces massive amounts of superoxide upon activation in a process called phagocyte oxidative burst. In addition to NOX2, superoxide is produced from various other cellular sources, such as from the mitochondria during cellular respiration and by other members of the NOX enzyme family [14]. However, it 
should be noted that during inflammation these superoxide producers are not nearly as efficient superoxide producers as the NOX2 complex.

In the current work we studied the role of SOD3 in collagen-induced arthritis (CIA) to understand whether the therapeutic effect of SOD3 on arthritis operates through attenuating the biological effects of the induced oxidative burst produced by the NOX2 complex. To avoid artifacts introduced by chemical inhibitors of NOX2 complex [1416] we used mice that genetically lack functional NOX2 complex. Thus we used wild-type $\left(\mathrm{Ncfl}^{+/+}\right)$and $\mathrm{Ncf1}$ mutated $\left(\mathrm{Ncfl}^{* / *}\right)$ mice on B10.Q background. These strains differ at only one SNP in the Ncf1 gene, which makes the $N c f 1^{* / *}$ strain unable to produce oxidative burst [13]. The mutated mouse is more susceptible to induced arthritis due to hyperactivated T cells [13], and also increased susceptibility to thioglycollate peritonitis has been reported in the Ncf1 knockout mouse [17]. Our results confirm the previously documented anti-inflammatory role of SOD3 and additionally, for the first time, we show that it can downregulate both CIA and peritonitis even in the absence of functional NOX2 complex and phagocyte oxidative burst.

\section{Materials and Methods}

2.1. Mice. The previously described Ncf1 ${ }^{m 1}$ (protein also called p47phox) mouse [18], which carries a point mutation globally and completely abolishing NOX2 complex derived ROS production, has been backcrossed onto the B10.Q background [13] and shown to contain only the causative mutation using a $10 \mathrm{k}$ SNP typing chip. The mice were housed under specific pathogen-free conditions in climatecontrolled environment and fed standard rodent chow and water ad libitum at Turku University Central Animal Facility. All experimental mice were sex- and age-matched, treatment groups were blinded, and experimental groups were mixed in cages in all experiments. The experiments were performed in accordance with the national and EU guidelines and the study was approved by the Oulu section of the national Animal Experiment Board (Eläinkoelautakunta, ELLA) with ethical approval numbers ESLH-2008-02873, ESLH-200807941, and ESAVI-0000497/041003/2011.

2.2. Viral Expression Vectors Used in the Study. Replication deficient adenoviral E1-partially-E3-deleted AdBglII vectors (developed from serotype Ad5) expressing rabbit SOD3 (Ade-SOD3) or bacterial $\beta$-galactosidase lacZ (Ade-LacZ) [4] were used in both in vitro and in vivo experiments.

2.3. Collagen-Induced Arthritis. Collagen-induced arthritis (CIA) was induced under isoflurane anesthesia by injecting $100 \mu \mathrm{g}$ rat type II collagen (purified from chondrosarcoma) [19] emulsified in complete Freund's adjuvant intradermally at the base of the tail. Arthritis was boosted day 19 with $50 \mu \mathrm{g}$ rat type II collagen emulsified in incomplete Freund's adjuvant intradermally at the base of the tail. Disease development was evaluated macroscopically three times a week before the booster immunization and daily after the boost [20]. One point was given for each swollen toe or joint and five points for a swollen ankle, each paw having the maximum of fifteen points.

In the arthritis experiments Ade-SOD3, Ade-LacZ (vector control), and PBS (injection control) were injected locally in the left front paw in $25 \mu \mathrm{L}$ injection volume containing 2,5 $\times 10^{8} \mathrm{PFU}$ virus. Injections were performed right after the booster immunization during the same anesthesia at day 19 , before the onset of clinically apparent arthritis.

2.4. Peritonitis. Peritonitis experiments were performed as described in [2]. Briefly, mice were pretreated i.p. with $0.5 \times 10^{9} \mathrm{PFU}$ Ade-SOD3, Ade-LacZ, or PBS three days before peritonitis induction with $5 \%$ proteose peptone (BD Difco, Sparks, MD, USA) and 10 ng IL- $1 \beta$ (R\&D Systems, Minneapolis, MN, USA) in $1 \mathrm{~mL}$ PBS. After 18 hours peritoneal infiltrating cells were collected with $10 \mathrm{~mL}$ icecold RPMI cell culture medium. Cells from the peritoneal lavage were counted and cytocentrifuged, slides were stained with Reastain Diff-Quick (Reagena, Toivala, Finland), and differential counting was performed under a standard light microscope. Experiments were pooled and the total cell numbers are presented as percentual increase from the PBS injection control. All peritonitis results were normalized to adjust the vector control group (Ade-LacZ) mean to 100.

2.5. Cell Culture and In Vitro Extracellular Oxidative Burst. COS-7 cells stably expressing all the essential components of the NOX2 complex, namely, Cybb (gp91phox), Cyba (p22phox), Ncf2 (p67phox), and Ncf1 (p47phox) were provided by Dr. Mary C. Dinauer, Indiana University, USA [21]. Cells were cultured in Dulbecco's complete medium (Gibco), 10\% fetal calf serum, and penicillin-streptomycin (Invitrogen, Paisley UK).

Extracellular superoxide production was quantified two days after transduction (MOI 4) with adenoviral constructs (Ade-SOD3 and Ade-LacZ) or medium control directly on the 96-well cell culture plate using an isoluminol-enhanced chemiluminescence method $[22,23]$. Briefly, the cells were washed with PBS, and $100 \mu \mathrm{L}$ of isoluminol buffer was added in each well. Isoluminol buffer contained isoluminol ( $10 \mu \mathrm{g} / \mathrm{mL}$, Sigma-Aldrich) and horse radish peroxidase-type II (4 U/mL, Sigma-Aldrich) dissolved in PBS with PMA $(200 \mathrm{ng} / \mathrm{mL}$, dissolved in DMSO, Sigma-Aldrich) and data collection was initiated immediately and followed at $37^{\circ} \mathrm{C}$ as produced luminescence signal (Tecan Infinite M200, Tecan, Männedorf, Switzerland) for 30 minutes. DMSO vehicle controls represent the nonstimulated background values. Representative data from the 15 -min time point is reported.

2.6. In Vitro Intracellular Oxidative Burst. Red blood cells were lysed from heparinized whole blood with hypotonic lysis buffer and leukocytes were surface stained with APC conjugated anti-Gr-1 (RB6-8C5) and eFluor 450 conjugated anti-CD11b (M1/70) antibodies (eBioscience). Cells were suspended in high-glucose D-MEM (Gibco) with antibiotics without FCS and incubated for $10 \mathrm{~min}$ at $37^{\circ} \mathrm{C}$ with $3 \mu \mathrm{M}$ dihydro-rhodamine 123 (DHR-123; Molecular Probes and 
Invitrogen Life Technologies) followed by $20 \mathrm{~min}$ activation at $37^{\circ} \mathrm{C}$ with $200 \mathrm{ng} / \mathrm{mL}$ PMA (Sigma-Aldrich). After oxidation by ROS, DHR-123 emits fluorescence upon excitation with the blue laser. The cells were washed into PBS and acquired on LSR II flow cytometer equipped with FACS Diva software (BD Biosciences). Live cells were gated on the cell type, and geometric means of respective populations were analyzed with Flowing Software (Cell Imaging Core, University of Turku).

2.7. In Vivo Oxidative Burst. Isoflurane anesthetized animals with equal arthritis scores were injected intraperitoneally with $20 \mathrm{mg} / \mathrm{kg}$ L-012 probe (Wako Chemicals, Germany) dissolved in PBS [24]. The luminescent signal was detected with IVIS 50 bioluminescent system (Xenogen, USA) that consists of an anesthesia unit built in a light tight chamber equipped with a CCD camera. Image acquisition and analysis were performed with Living Image software (Xenogen).

2.8. Detection of Ade-SOD3 from the Injected Paws by RT$P C R$. RNA was isolated from the treated paws collected d25 according to the manufacturer's instructions with TRI Reagent (Sigma-Aldrich). Glass homogenizers were used to homogenize and separate the soft tissue from the bone. The isolated RNA was DNAse treated with deoxyribonuclease I (Fermentas) in the presence of Ribolock RNase Inhibitor (Fermentas). The RNA was used for reverse transcription reaction performed with Revert-Aid M-MULV (Fermentas). The acquired cDNA was subjected to Q-PCR with Ade-SOD3 (fw: GTG TGC TCC TGC CTG CTC, rev: CTG CTC CAC CGT GTC TGA G) and $\beta$-actin specific primers (fw: CTA AGG CCA ACC GTG AAA AG, rev: ACC AGA GGC ATA CAG GGA CA), and the gene expression level was analyzed using SYBR Green PCR Master Mix (Applied Biosystems), iCycler iQ Multicolor Real-Time PCR Detection System (Bio-Rad), and the iCycler (version 3.1) software. Ade-SOD signal was normalized against $\beta$-actin expression levels and the results are reported as fold change from the Ade-LacZ group mean denoted as 1 .

2.9. Statistics. Quantitative data are expressed as \pm SEM. Statistical significance was analyzed by using two-tailed Student's $t$-test or if more than two groups were analyzed one-way ANOVA with LSD post hoc analysis was run using IBM SPSS Statistics 19 software (SPSS Inc.), $P<0.05$ is considered as statistically significant.

\section{Results}

3.1. Ade-SOD3 Produced Enzymatically Active SOD3 In Vitro. Adenoviral SOD3 gene delivery reduced the superoxide predominant ROS signal 37\% compared to the Ade-LacZ control virus when investigated two days after transduction with Ade-SOD3 and Ade-LacZ viruses (Figure 1).

3.2. SOD3 Downregulated Arthritis. Mice started to develop mild, clinically apparent arthritis at day 20 and made a full response, mean arthritis scores reaching 7 (out of the

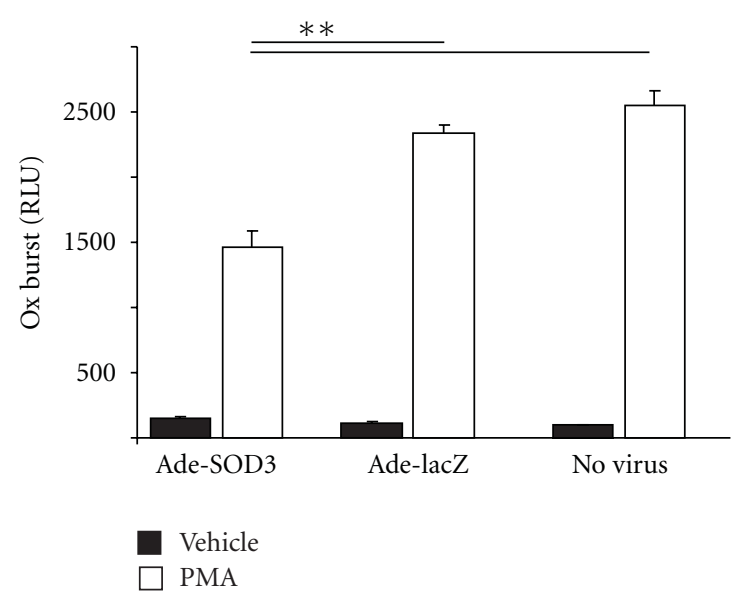

FIgure 1: The amount of extracellular ROS is reduced by SOD3. Adenoviral vector coding for SOD3 (Ade-SOD3) significantly reduced the amount of extracellular ROS produced by COS cells expressing all functional components of the NOX2 complex, when compared to the Ade-LacZ control virus.

maximum of 15) in Ade-Laz treated and 3 points in AdeSOD3-treated mice some days later. The mean disease score in the treated paws was lower in the Ade-SOD3-treated group when compared to the Ade-LacZ-injected control group, and the difference reached statistical significance at d26 (Figure 2(a)). The treatment effect was only seen in the treated paw, while there were no differences between the treatment groups in sum scores of the three untreated paws (see Supplementary Figure 1(a) in supplementary material available online at doi: 10.1155/2012/730469) highlighting the local character of the used gene therapy vector expressing SOD3.

Both virus vector treated groups showed elevated arthritis scores in the treated paws when compared to the PBSinjected control paws in the injection control group. This difference is illustrated in Figure 2(b), where Ade-LacZtreated paws are shown to have larger increase in arthritis score than the Ade-SOD3-treated paws.

3.3. SOD3 Downregulated Arthritis in the Absence of Functional NOX2 Complex. Starting from d20 the $N c f 1^{* / *}$ mice started to develop arthritis with significantly higher mean score than the wild-type mice. Interestingly, the mutated mice without functional NOX2 complex derived superoxide production also responded to Ade-SOD3 treatment. AdeSOD3-treated paws of the $N c f 1^{* / *}$ mice had significantly lower mean disease score than the Ade-LacZ-treated vector control paws at days 24 and 25 after arthritis induction. Similarly to the wild-type mice, the difference in the mean arthritis score in NOX2 complex deficient mice was observed when SOD3 was highly expressed from the adenoviral vector (Figure 3(a)) [4]. No differences were observed between the treatment groups in the sum scores of the untreated paws (Supplementary Figure 1(b)) again supporting the local character of the immunomodulatory effect of Ade-SOD3 treatment. 


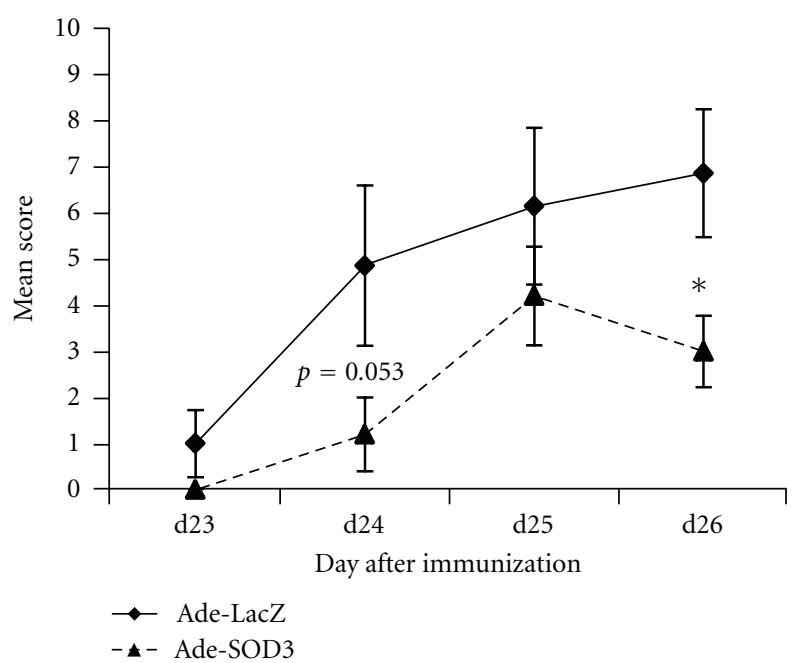

(a)

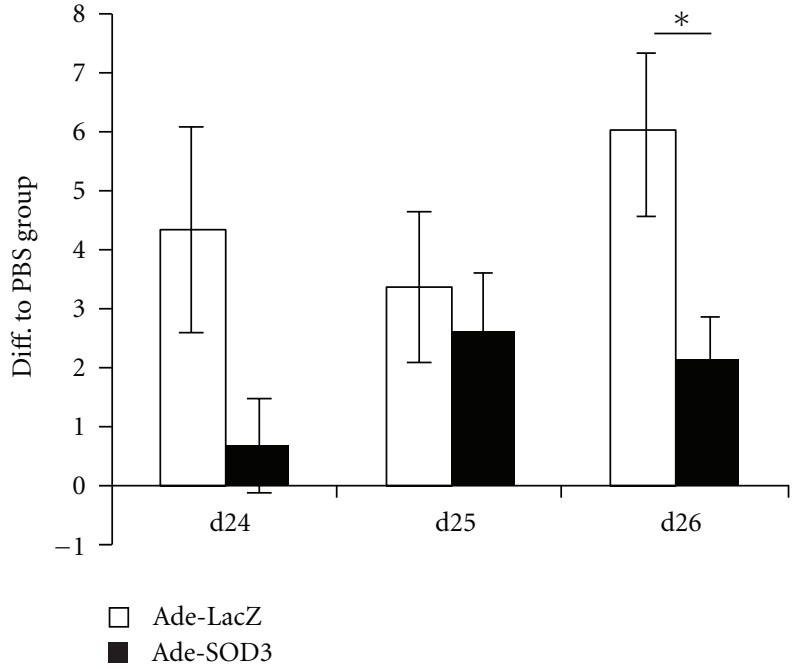

(b)

FIgURE 2: SOD3 downregulated arthritis in wild-type B10.Q mice. Adenovirally expressed SOD3 reduced arthritis severity in the treated paws (a) in wild-type B10.Q mice (Ade-SOD3 $n=6$, Ade-lacZ $n=7$ ). When compared to the PBS-treated injection control ( $n=8$ ) group, Ade-SOD3-treated mice had less increase in the arthritis score in the treated paw than the Ade-LacZ-treated control mice (b).

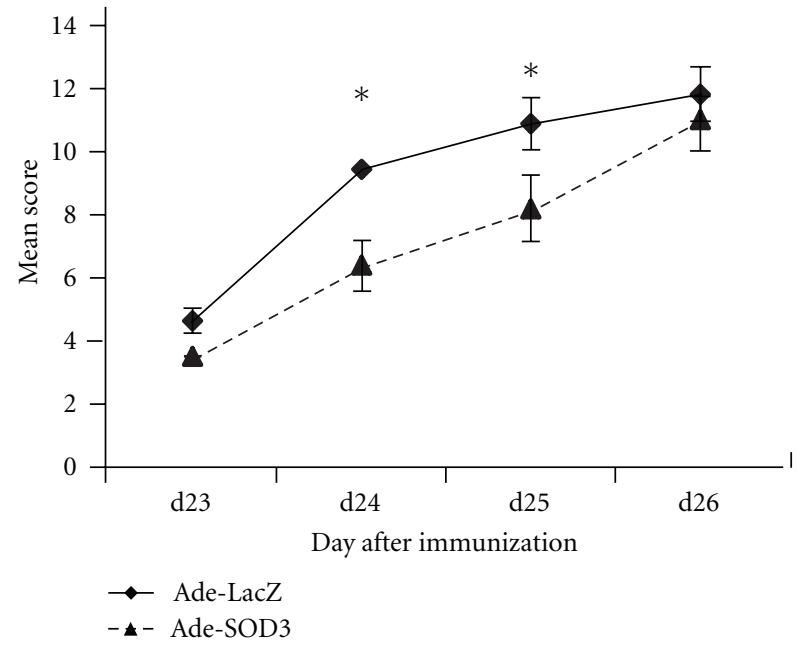

(a)

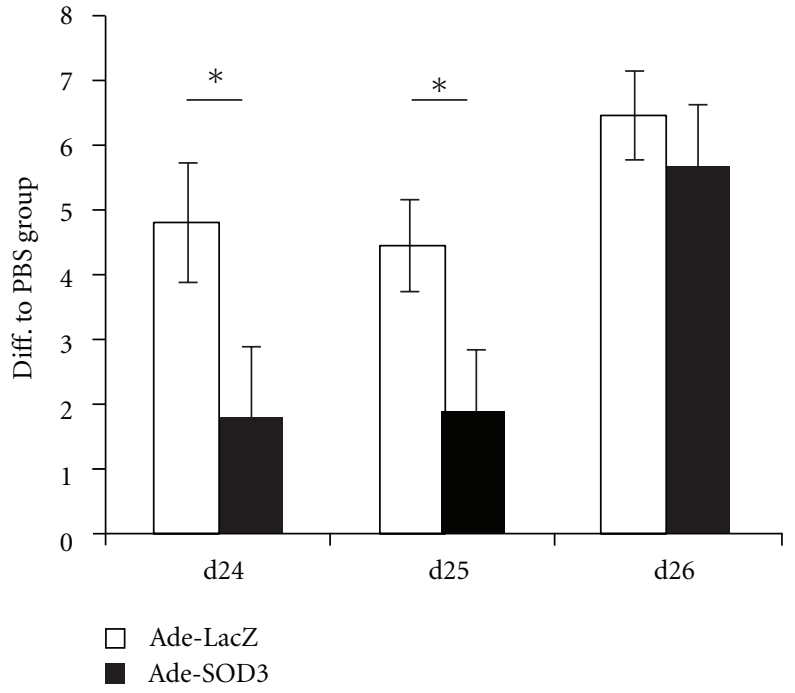

(b)

FIgURE 3: SOD3 downregulated arthritis in the absence of functional NOX2 complex. Arthritis severity was significantly reduced in AdeSOD3-treated paws of mice lacking functional oxidative burst when compared to the control vector-treated paws (a). Shown is pooled data from two independent experiments (Ade-SOD3 $n=22$, Ade-lacZ $n=23$ ). The difference in arthritis score in the treated paw between the PBS-treated control mice $(n=10)$ and Ade-SOD3-treated mice was smaller than the difference between PBS-treated and Ade-LacZ-treated mice at days 24 and 25 (b).

Similarly to the wild-type mice, Ade-SOD3 treatment in $N c f 1^{* / *}$ paws induced significantly smaller difference between the virus-treated and PBS-injected paws than Ade-LacZ virus injection (significant $\mathrm{d} 24$ and $\mathrm{d} 25$ after immunization), again confirming the arthritis limiting effect of SOD3 (Figure 3(b)). The experiment was repeated and as the experiments were well reproduced, data from both experiments was combined for analysis.
3.4. SOD3 Limited Peritonitis Both in the Presence and Absence of Phagocyte Oxidative Burst. SOD3 expression significantly reduced the number of peritoneal infiltrating cells in proteose peptone and IL-1 $\beta$ induced peritonitis in wild type mice (Figure 4(a)). The decrease in infiltrating leukocytes was mainly due to a lowered number of infiltrating macrophages (Figure 4(b)), which well corresponds with the macrophage phase of peritonitis taking place three days 


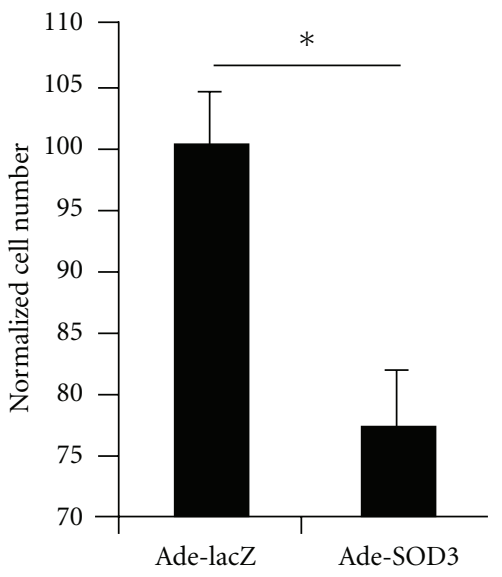

(a)

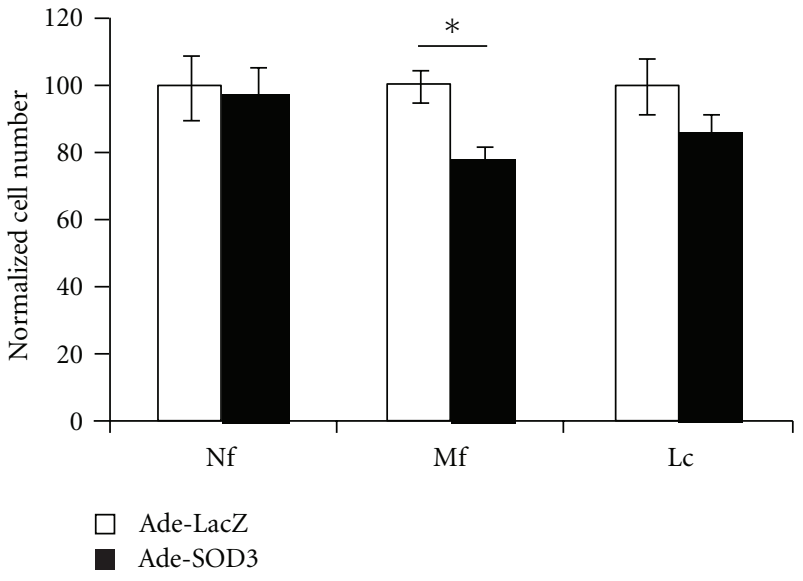

(b)

FIGURE 4: Peritonitis is limited by SOD3. Ade-SOD3-treated mice had less peritoneal infiltrating cells after peritonitis induction with proteose peptone and IL-1 $\beta$ than Ade-LacZ-treated control mice (a) $(n=5)$. The reduction in cell number was mainly due to diminished peritoneal macrophage population $(\mathrm{b})(\mathrm{Nf}=$ neutrophil, $\mathrm{Mf}=$ macrophage, $\mathrm{Lc}=$ lymphocyte).

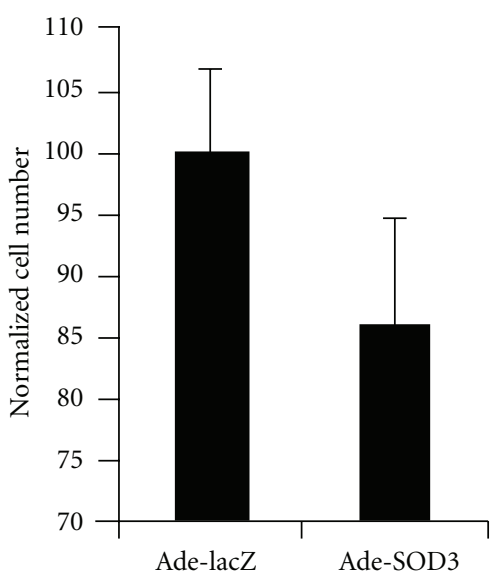

(a)

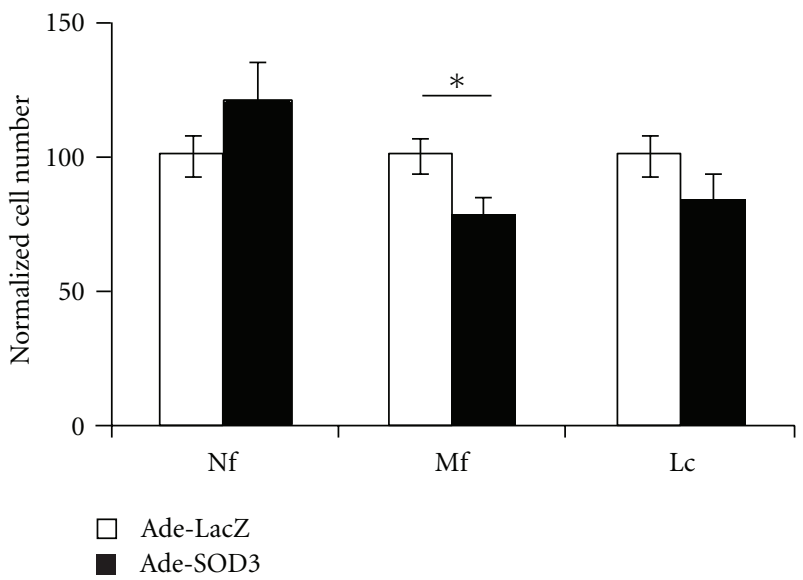

(b)

FIGURe 5: Peritonitis is limited by SOD3 in the absence of functional NOX2 complex. Similarly to wild-type mice, Ade-SOD3-treated Ncf1*/* mice had less peritoneal infiltrating macrophages than the Ade-LacZ-treated vector controls in the proteose peptone and IL- $1 \beta$-induced peritonitis model (a). Normalized total cell numbers (b) show significant decrease in infiltrating macrophage population. Shown is pooled data from two independent experiments $(n=10)(\mathrm{Nf}=$ neutrophil, $\mathrm{Mf}=$ macrophage, Lc $=$ lymphocyte $)$.

after virus injection and 18 hours after peritonitis induction. Both virus-treated groups had more infiltrating cells than the PBS-injected control mice.

Similarly as in the wild-type mice Ade-SOD3 was shown to reduce the number of infiltrating cells in the peritoneal cavity of $\mathrm{Ncfl}^{* / *}$ mice when compared to Ade-LacZ-treated mice. The difference was due to a significantly lowered number of infiltrating macrophages in the Ade-SOD3treated mice (Figures 5(a) and 5(b)).

\subsection{Ncf $1^{* / *}$ Mice Lack Oxidative Burst In Vivo and In Vitro.} In the wild-type mouse both severely inflamed paws (right hind leg and right front paw) emitted strong ROS-induced L-012 luminescence signal, while in spite of the severe inflammation, no luminescent signal was detected from the
$N c f 1^{* / *}$ mice (severe arthritis with arthritis score of 15 in both front paws and milder symptoms in the left hind leg) (Figure 6(a)). The intraperitoneal L-012 injection site emitted similar background signal in both genotypes.

Monocytes (CD11b-POS, Gr-1-LO, or Gr-1-NEG) and granulocytes (Gr-1-HI, CD11b-POS) from $\mathrm{Ncfl}^{* / *}$ mice were unable to generate efficient ROS production upon PMA stimulation, while phagocytes from the wild-type animals responded to PMA stimulation and induced significant increase in DHR-123 derived fluorescence signal detected by flow cytometry (Figures 6(c) and 6(d)).

3.6. Ade-SOD3 Expression In Vivo. Quantitative RT-PCR revealed a A 5.5-fold (FC, fold change) expression of adenovirally produced SOD3 mRNA in the Ade-SOD3-treated joints 


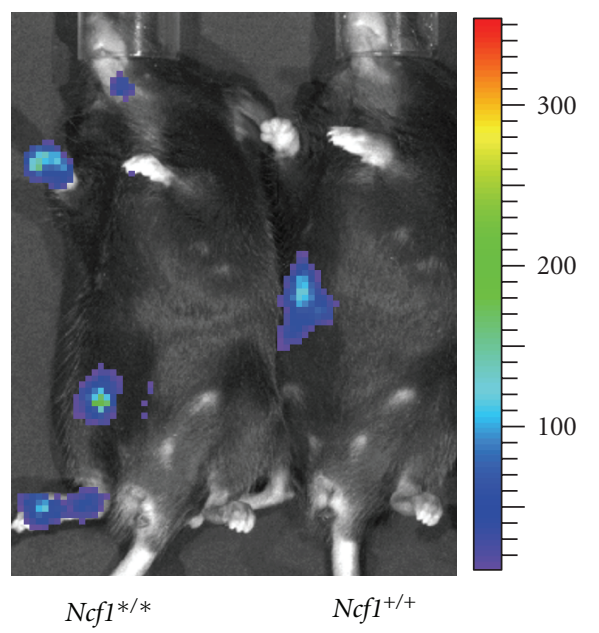

(a)

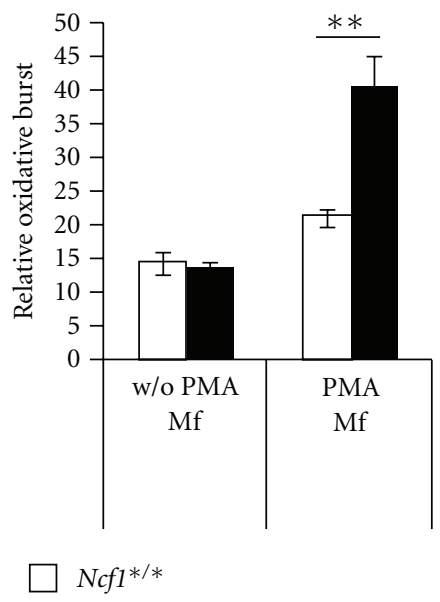

(c)

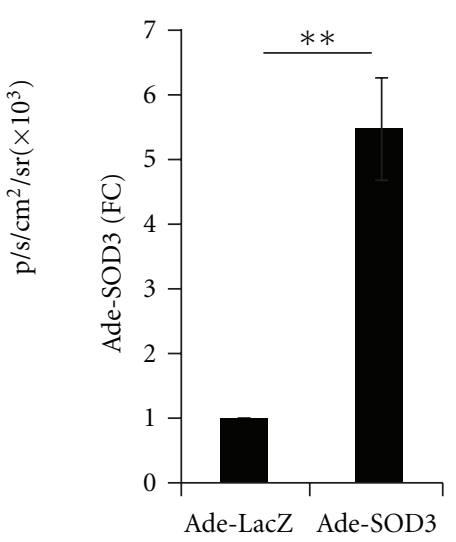

(b)

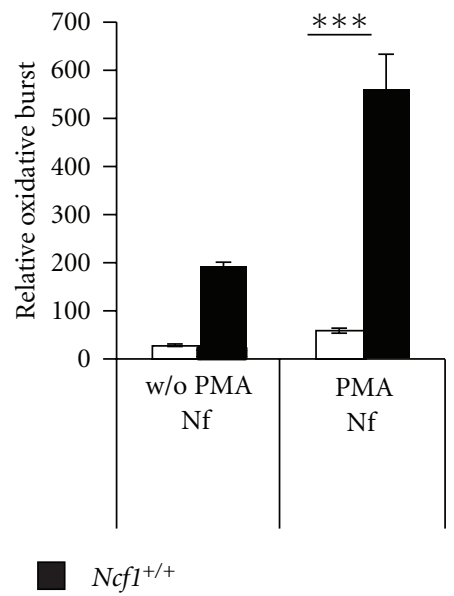

(d)

FIGURE 6: No oxidative burst was detected from the Ncf1 mutated mice. In vivo imaging with the ROS sensitive L-012 probe revealed a bright luminescent signal from the inflamed wild-type paws with CIA, while no signal was detected from $N c f 1^{* / *}$ mice with comparable arthritis severity (a). Imaging was reproduced in two independent experiments with similar results. Expression of SOD3 by Ade-SOD3 expression vector in the treated paws was confirmed by Q-RT-PCR ( $n=7$ /group) (b). Ex vivo oxidative burst was measured from phagocytes from $N c f 1^{* / *}(n=5)$ and wild-type mice $(n=6)$ by using DHR-123 assay with and without (w/o) PMA stimulation. Neither macrophages (c) nor neutrophils (d) from the $N c f 1^{* / *}$ mice were able to exert a measureable oxidative burst.

collected at $\mathrm{d} 25$ when compared to the Ade-LacZ-treated vector control paws (Figure 6(b)).

\section{Discussion}

SOD3 is an enzyme that has been shown to give rise to therapeutic responses in damaged tissues such as reduced ischemia-reperfusion injury [25], arthritis [7], peritonitis [2], hind limb injury [3], and lung injury [6] models. These tissue healing promoting and anti-inflammatory effects induced by SOD3 are accompanied by reduced macrophage infiltration [2], inhibition of oxidative fragmentation of the extracellular matrix matrix [26], decreased apoptosis [4, 5], and enhanced cell proliferation [27]. The beneficial effects of SOD3 are mostly explained by its antioxidant properties and reduction of oxidative stress in the injured tissues. However, in this report we show that overexpression of SOD3 downregulated inflammation even in the absence of phagocyte oxidative burst, thus highlighting the capacity of SOD3 to affect cellular processes independently of NOX2 complex' superoxide production.

The adenoviral gene expression system used in this work reaches its maximal expression around three days after the injection, after which the vector is eliminated by the immune system and the expression of the transgene slowly decreases to undetectable levels 14 days after the initial injection [4]. We confirmed the presence of virally delivered SOD3 mRNA in the paws $\mathrm{d} 25$ and thus confirmed that the decrease in arthritis severity coincided with substantial adenovirus driven SOD3 expression. Similarly, pretreating the mice three 
days before induction of peritonitis allowed us to analyze the effect of SOD3 during substantial SOD3 expression in the peritoneal cavity.

The percentual treatment effect in both inflammation models was comparable with the effect previously obtained with transgenic SOD3 overexpression in pulmonary emphysema [26]. Similarly, the degree of SOD3induced macrophage infiltration inhibition in peritonitis was similar as reported previously [2]. Even though SOD3 is an important local regulator of the acute inflammatory reaction, it is obvious that there are a number of other factors affecting inflammation severity in vivo.

The physiological function of SOD3 is to dismutate extracellular superoxide into hydrogen peroxide. When the NOX2 complex is not functional, there are still a number of other enzymes and enzyme complexes producing superoxide in the inflamed tissue. NOX1 has been reported to worsen hyperoxia-induced acute lung injury in mice [28], and NOX4 has been suggested to stimulate microglial IL-6 expression [29] and to hamper neurodegeneration after poststroke ischemia reperfusion injury [30]. However, only NOX2 is abundantly expressed on phagocytes and thus recruited to inflammatory foci. Other NOX family members are expressed on other cell types than phagocytes and their ROS production is not upregulated during inflammation.

In addition to the NOX family enzymes, superoxide is also produced during mitochondrial respiration at levels corresponding to the general metabolic rate in the tissue. Mitochondrial superoxide has also been linked to TLR2/4 signaling [31] and is also suggested as a pathologic mechanism in tissue injury [32]. All these superoxide generating processes, however, cannot compensate for the massive production of ROS by the NOX2 complex during inflammation as the arthritic paws of $N c f 1^{* / *}$ mice emitted no detectable luminescence signal when probed with L-012 dye (Figure 6(a)). L-012 reacts with any radical to produce light and cannot be used to distinguish between superoxide and hydrogen peroxide in vivo.

As a tool to study the NOX2 complex dependency of SOD3, we used Ncf1 mutated mice, in which a splice site point mutation abolishes the production of NCF1 protein leading to complete loss of NOX2 complex derived oxidative burst. In line with previous reports, arthritis severity was significantly higher in $N c f 1^{* / *}$ animals when compared to B10.Q wild-type mice [13]. Mutated mice also developed arthritis quicker after the booster allowing us to work with a more homogenous and extremely well reproducible disease model. Better arthritis synchronization in the mutated mouse model together with larger treatment groups resulted in less variability and increased statistical power in data analysis. In proteose peptone-induced peritonitis we observed no difference between the genotypes. The use of Ncfl mutant mice allowed us to avoid pit falls associated with the use of chemical NOX2 complex inhibitors such as DPI and apocynin, which are not specific for the NOX2 complex, do not provide full suppression of superoxide production and additionally profoundly affect various other cellular processes [14-16].
In both arthritis and peritonitis, adenoviral gene expression vectors locally enhanced inflammation. The arthritis enhancing effect was restricted to the treated paw, as the virus-injected groups did not differ from the PBS-injected control group when their nontreated paws were analyzed. This is in line with previous reports where intra-articular injections of adenoviral gene expression vectors have been shown to induce increased paw swelling and elevated levels of inflammation mediators [33, 34]. Intravenous injection route has not given rise to enhanced arthritis [35-37], but has triggered liver inflammation; liver being the primary target of systemically administered adenoviral vectors [35]. The immunogenicity of the adenoviral gene expression systems is well documented in the literature [38-40].

ROS regulate a number of physiological and diseaserelated pathways in humans. SOD3 polymorphisms are associated with COPD [41], coronary artery disease, myocardial infarction [42] as well as acute lung injury and related mortality [43]. Additionally, SOD3 has been reported to be downregulated in thyroid cancer tumors [44]. Both SOD3 and Ncf1 are highly conserved ROS regulators [45]. There is also genetic [46] and functional [47] evidence linking Ncf1 to human diseases.

\section{Conclusions}

We report that SOD3 limits inflammation in CIA and peritonitis both in the presence and in the absence of phagocyte oxidative burst. The anti-inflammatory function of SOD3 is not compromised by the lack of functionality of the NOX2 complex as both $\mathrm{Ncf1}^{+/+}$and $\mathrm{Ncf1}{ }^{* / *}$ mice develop milder inflammation when treated with SOD3. Thus, we conclude that the anti-inflammatory effect of SOD3 is not dependent on superoxide produced by the NOX2 complex derived phagocyte oxidative burst and thus acts via other signaling pathways.

\section{Acknowledgments}

This project has received financial support from Academy of Finland, Sigrid Juselius Foundation, Nordic Centre of Excellence in disease genetics, Maire Lisko foundation, and the European Union Grant Masterswitch (HEALTH-F22008-223404). Mary C. Dinauer (Indiana University) is acknowledged for kindly providing us with the COS-7 cells used in in vitro experiments. The authors want to thank Seija Lindqvist, Toni Ilo, and Tiina Kyrölä for expert animal care.

\section{References}

[1] J. M. McCord and I. Fridovich, "Superoxide dismutase. An enzymic function for erythrocuprein (hemocuprein)," Journal of Biological Chemistry, vol. 244, no. 22, pp. 6049-6055, 1969.

[2] J. P. Laurila, L. E. Laatikainen, M. D. Castellone, and M. O. Laukkanen, "SOD3 reduces inflammatory cell migration by regulating adhesion molecule and cytokine expression," PLoS One, vol. 4, no. 6, Article ID e5786, 2009.

[3] J. P. Laurila, M. D. Castellone, A. Curcio et al., "Extracellular superoxide dismutase Is a growth regulatory mediator of tissue 
injury recovery," Molecular Therapy, vol. 17, no. 3, pp. 448454, 2009.

[4] M. O. Laukkanen, P. Leppanen, P. Turunen, T. Tuomisto, J. Naarala, and S. Yla-Herttuala, "EC-SOD gene therapy reduces paracetamol-induced liver damage in mice," Journal of Gene Medicine, vol. 3, no. 4, pp. 321-325, 2001.

[5] L. E. Laatikainen, M. Incoronato, M. D. Castellone, J. P. Laurila, M. Santoro, and M. O. Laukkanen, "SOD3 decreases ischemic injury derived apoptosis through phosphorylation of Erk1/2, Akt, and Foxo3a," PLoS One, vol. 6, no. 8, Article ID e24456, 2011.

[6] R. J. Folz, A. M. Abushamaa, and H. B. Suliman, "Extracellular superoxide dismutase in the airways of transgenic mice reduces inflammation and attenuates lung toxicity following hyperoxia," Journal of Clinical Investigation, vol. 103, no. 7, pp. 1055-1066, 1999.

[7] S. Iyama, T. Okamoto, T. Sato et al., "Treatment of murine collagen-induced arthritis by ex vivo extracellular superoxide dismutase gene transfer," Arthritis and Rheumatism, vol. 44, no. 9, pp. 2160-2167, 2001.

[8] D. Salvemini, E. Mazzon, L. Dugo et al., "Amelioration of joint disease in a rat model of collagen-induced arthritis by M40403, a superoxide dismutase mimetic," Arthritis and Rheumatism, vol. 44, no. 12, pp. 2909-2921, 2001.

[9] A. D. Ross, N. K. Banda, M. Muggli, and W. P. Arend, "Enhancement of collagen-induced arthritis in mice genetically deficient in extracellular superoxide dismutase," Arthritis and Rheumatism, vol. 50, no. 11, pp. 3702-3711, 2004.

[10] E. A. Ostrakhovitch and I. B. Afanas'ev, "Oxidative stress in rheumatoid arthritis leukocytes: suppression by rutin and other antioxidants and chelators," Biochemical Pharmacology, vol. 62, no. 6, pp. 743-746, 2001.

[11] J. Cedergren, T. Forslund, T. Sundqvist, and T. Skogh, "Intracellular oxidative activation in synovial fluid neutrophils from patients with rheumatoid arthritis but not from other arthritis patients," Journal of Rheumatology, vol. 34, no. 11, pp. 2162-2170, 2007.

[12] P. Olofsson, J. Holmberg, J. Tordsson, S. Lu, B. Åkerström, and R. Holmdahl, "Positional identification of $\mathrm{Ncfl}$ as a gene that regulates arthritis severity in rats," Nature Genetics, vol. 33, no. 1, pp. 25-32, 2003.

[13] M. Hultqvist, P. Olofsson, J. Holmberg, B. T. Bäckström, J. Tordsson, and R. Holmdahl, "Enhanced autoimmunity, arthritis, and encephalomyelitis in mice with a reduced oxidative burst due to a mutation in the Ncf1 gene," Proceedings of the National Academy of Sciences of the United States of America, vol. 101, no. 34, pp. 12646-12651, 2004.

[14] K. Bedard and K. H. Krause, "The NOX family of ROSgenerating NADPH oxidases: physiology and pathophysiology," Physiological Reviews, vol. 87, no. 1, pp. 245-313, 2007.

[15] E. Aldieri, C. Riganti, M. Polimeni et al., "Classical inhibitors of NOX NAD(P)H oxidases are not specific," Current Drug Metabolism, vol. 9, no. 8, pp. 686-696, 2008.

[16] F. L. Van De Veerdonk, S. P. Smeekens, L. A. B. Joosten et al., "Reactive oxygen species-independent activation of the IL- $1 \beta$ inflammasome in cells from patients with chronic granulomatous disease," Proceedings of the National Academy of Sciences of the United States of America, vol. 107, no. 7, pp. 3030-3033, 2010.

[17] S. H. Jackson, J. I. Gallin, and S. M. Holland, “The p47(phox) mouse knock-out model of chronic granulomatous disease," Journal of Experimental Medicine, vol. 182, no. 3, pp. 751-758, 1995.
[18] C. K. Huang, L. Zhan, M. O. Hannigan, Y. Ai, and T. L. Leto, "P47(phox)-deficient NADPH oxidase defect in neutrophils of diabetic mouse strains, C57BL/6J-m db/db and db/+," Journal of Leukocyte Biology, vol. 67, no. 2, pp. 210-215, 2000.

[19] B. D. Smith, G. R. Martin, and E. J. Miller, "Nature of the collagen synthesized by a transplanted chondrosarcoma," Archives of Biochemistry and Biophysics, vol. 166, no. 1, pp. 181-186, 1975.

[20] R. Holmdahl, R. Bockermann, J. Bäcklund, and H. Yamada, "The molecular pathogenesis of collagen-induced arthritis in mice-a model for rheumatoid arthritis," Ageing Research Reviews, vol. 1, no. 1, pp. 135-147, 2002.

[21] M. O. Price, L. C. McPhail, J. D. Lambeth, C. H. Han, U. G. Knaus, and M. C. Dinauer, "Creation of a genetic system for analysis of the phagocyte respiratory burst: high-level reconstitution of the NADPH oxidase in a nonhematopoietic system," Blood, vol. 99, no. 8, pp. 2653-2661, 2002.

[22] H. Lundqvist and C. Dahlgren, "Isoluminol-enhanced chemiluminescence: a sensitive method to study the release of superoxide anion from human neutrophils," Free Radical Biology and Medicine, vol. 20, no. 6, pp. 785-792, 1996.

[23] C. Dahlgren and A. Karlsson, "Respiratory burst in human neutrophils," Journal of Immunological Methods, vol. 232, no. 1-2, pp. 3-14, 1999.

[24] A. Kielland, T. Blom, K. S. Nandakumar, R. Holmdahl, R. Blomhoff, and H. Carlsen, "In vivo imaging of reactive oxygen and nitrogen species in inflammation using the luminescent probe L-012," Free Radical Biology and Medicine, vol. 47, no. 6, pp. 760-766, 2009.

[25] P. O. Sjoquist, L. Carlsson, G. Jonason, S. L. Marklund, and T. Abrahamsson, "Cardioprotective effects of recombinant human extracellular-superoxide dismutase type $\mathrm{C}$ in rat isolated heart subjected to ischemia and reperfusion," Journal of Cardiovascular Pharmacology, vol. 17, no. 4, pp. 678-683, 1991.

[26] H. Yao, G. Arunachalam, J. W. Hwang et al., "Extracellular superoxide dismutase protects against pulmonary emphysema by attenuating oxidative fragmentation of ECM," Proceedings of the National Academy of Sciences of the United States of America, vol. 107, no. 35, pp. 15571-15576, 2010.

[27] R. L. Auten, M. A. O'Reilly, T. D. Oury, E. Nozik-Grayck, and M. H. Whorton, "Transgenic extracellular superoxide dismutase protects postnatal alveolar epithelial proliferation and development during hyperoxia," American Journal of Physiology, vol. 290, no. 1, pp. L32-L40, 2006.

[28] S. Carnesecchi, C. Deffert, A. Pagano et al., "NADPH oxidase1 plays a crucial role in hyperoxia-induced acute lung injury in mice," American Journal of Respiratory and Critical Care Medicine, vol. 180, no. 10, pp. 972-981, 2009.

[29] B. Li, K. Bedard, S. Sorce, B. Hinz, M. Dubois-Dauphin, and K. H. Krause, "NOX4 expression in human microglia leads to constitutive generation of reactive oxygen species and to constitutive il-6 expression," Journal of Innate Immunity, vol. 1, no. 6, pp. 570-581, 2009.

[30] C. Kleinschnitz, H. Grund, K. Wingler et al., "Post-stroke inhibition of induced NADPH Oxidase type 4 prevents oxidative stress and neurodegeneration," PLoS Biology, vol. 8, no. 9, Article ID e1000479, 2010.

[31] A. P. West, I. E. Brodsky, C. Rahner et al., "TLR signalling augments macrophage bactericidal activity through mitochondrial ROS," Nature, vol. 472, no. 7344, pp. 476-480, 2011.

[32] T. Mitchell, H. Saba, J. Laakman, N. Parajuli, and L. A. MacMillan-Crow, "Role of mitochondrial-derived oxidants in 
renal tubular cell cold-storage injury," Free Radical Biology and Medicine, vol. 49, no. 8, pp. 1273-1282, 2010.

[33] C. H. Le, A. G. Nicolson, A. Morales, and K. L. Sewell, "Suppression of collagen-induced arthritis through adenovirusmediated transfer of a modified tumor necrosis factor $\alpha$ receptor gene," Arthritis and Rheumatism, vol. 40, no. 9, pp. 1662-1669, 1997.

[34] J. M. Woods, K. J. Katschke, M. V. Volin et al., "IL-4 adenoviral gene therapy reduces inflammation, proinflammatory cytokines, vascularization, and bony destruction in rat adjuvant-induced arthritis," Journal of Immunology, vol. 166, no. 2, pp. 1214-1222, 2001.

[35] E. Quattrocchi, M. J. Dallman, A. P. Dhillon, A. Quaglia, G. Bagnato, and M. Feldmann, "Murine IL-10 gene transfer inhibits established collagen-induced arthritis and reduces adenovirus-mediated inflammatory responses in mouse liver," Journal of Immunology, vol. 166, no. 10, pp. 5970-5978, 2001.

[36] P. F. Sumariwalla, P. Jin, J. Zhang et al., "Antagonism of the human epidermal growth factor receptor family controls disease severity in murine collagen-induced arthritis," Arthritis and Rheumatism, vol. 58, no. 10, pp. 3071-3080, 2008.

[37] F. Apparailly, C. Verwaerde, C. Jacquet, C. Auriault, J. Sany, and C. Jorgensen, "Adenovirus-mediated transfer of viral IL10 gene inhibits murine collagen-induced arthritis," Journal of Immunology, vol. 160, no. 11, pp. 5213-5220, 1998.

[38] T. Yamaguchi, K. Kawabata, N. Koizumi et al., "Role of MyD88 and TLR9 in the innate immune response elicited by serotype 5 adenoviral vectors," Human Gene Therapy, vol. 18, no. 8, pp. 753-762, 2007.

[39] Z. Xu, J. Tian, J. S. Smith, and A. P. Byrnes, "Clearance of adenovirus by Kupffer cells is mediated by scavenger receptors, natural antibodies, and complement," Journal of Virology, vol. 82, no. 23, pp. 11705-11713, 2008.

[40] D. M. Appledorn, A. McBride, S. Seregin et al., "Complex interactions with several arms of the complement system dictate innate and humoral immunity to adenoviral vectors," Gene Therapy, vol. 15, no. 24, pp. 1606-1617, 2008.

[41] I. C. Sørheim, D. L. Demeo, G. Washko et al., "Polymorphisms in the superoxide dismutase-3 gene are associated with emphysema in COPD," COPD, vol. 7, no. 4, pp. 262-268, 2010.

[42] T. B. Grammer, W. Renner, M. M. Hoffmann et al., "SOD3 R231G polymorphism associated with coronary artery disease and myocardial infarction. The Ludwigshafen Risk and Cardiovascular Health (LURIC) study," Free Radical Research, vol. 43, no. 7, pp. 677-684, 2009.

[43] J. J. Arcaroli, J. E. Hokanson, E. Abraham et al., "Extracellular superoxide dismutase haplotypes are associated with acute lung injury and mortality," American Journal of Respiratory and Critical Care Medicine, vol. 179, no. 2, pp. 105-112, 2009.

[44] L. E. Laatikainen, M. D. Castellone, A. Hebrant et al., "Extracellular superoxide dismutase is a thyroid differentiation marker down-regulated in cancer," Endocrine-Related Cancer, vol. 17, no. 3, pp. 785-796, 2010.

[45] D. Colinet, D. Cazes, M. Belghazi, J.-L. Gatti, and M. Poirié, "Extracellular superoxide dismutase in insects: characterization, function, and interspecific variation in parasitoid wasp venom," Journal of Biological Chemistry, vol. 286, no. 46, pp. 40110-40121, 2011.

[46] L. M. Olsson, A. Nerstedt, A.-K. Lindqvist et al., "Copy number variation of the gene NCF1 is associated with rheumatoid arthritis," Antioxidants and Redox Signaling, vol. 16, no. 1, pp. 71-78, 2012.

[47] M. D. Kraaij, N. D. L. Savage, S. W. Van Der Kooij et al., "Induction of regulatory $\mathrm{T}$ cells by macrophages is dependent on production of reactive oxygen species," Proceedings of the National Academy of Sciences of the United States of America, vol. 107, no. 41, pp. 17686-17691, 2010. 


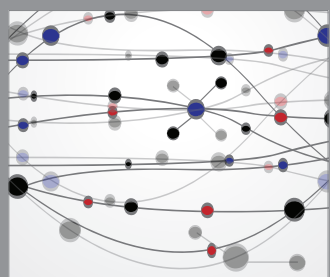

The Scientific World Journal
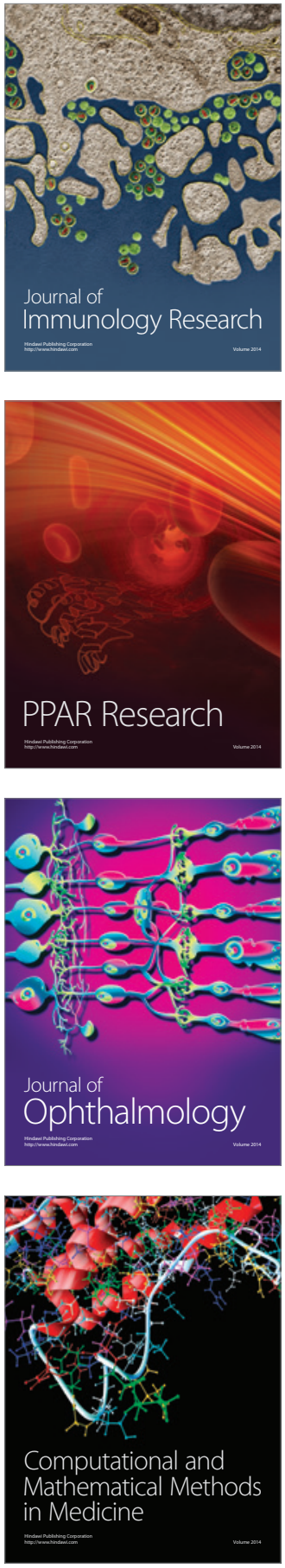

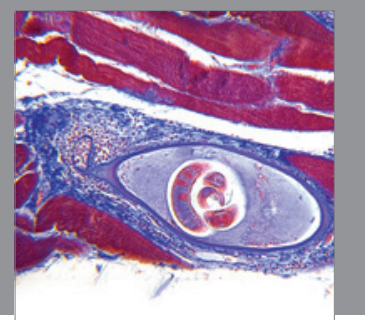

Gastroenterology

Research and Practice
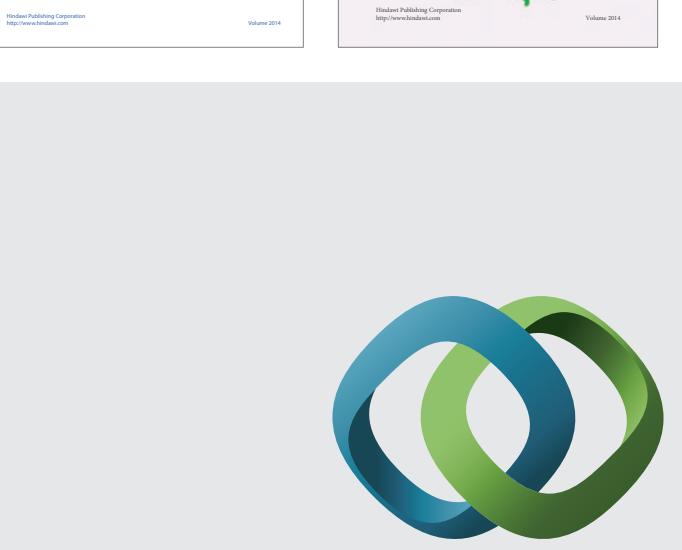

\section{Hindawi}

Submit your manuscripts at

http://www.hindawi.com
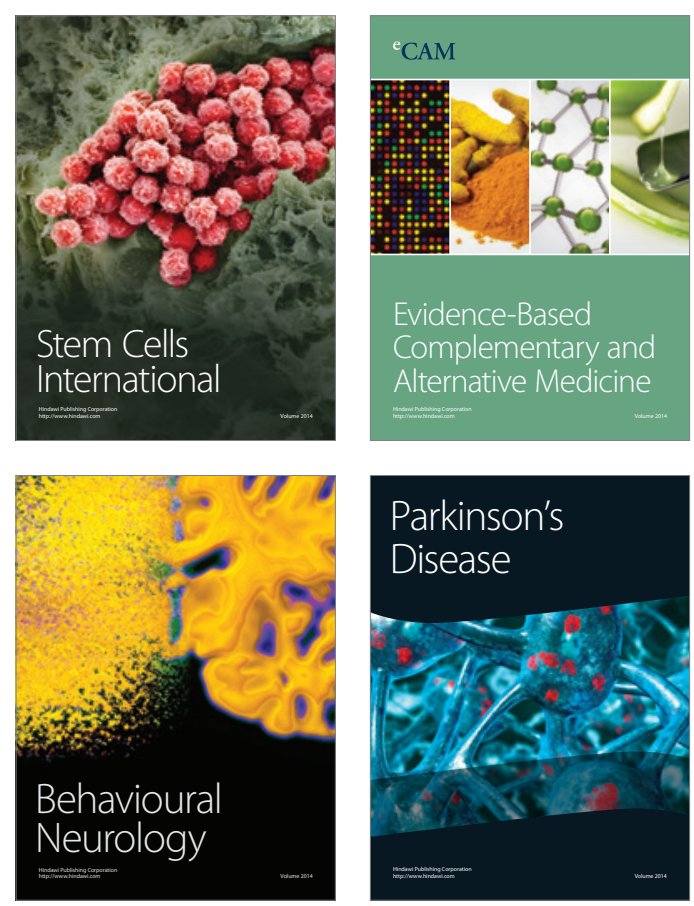

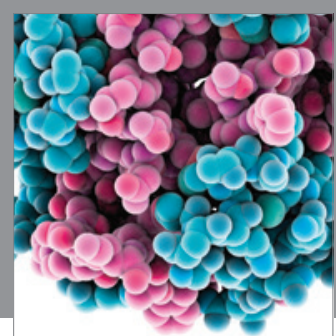

Journal of
Diabetes Research

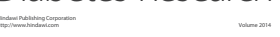

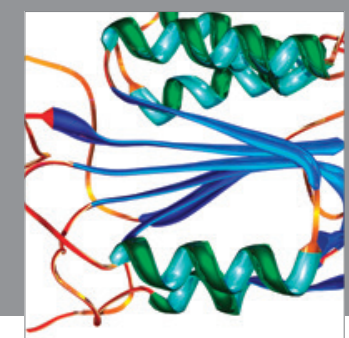

Disease Markers
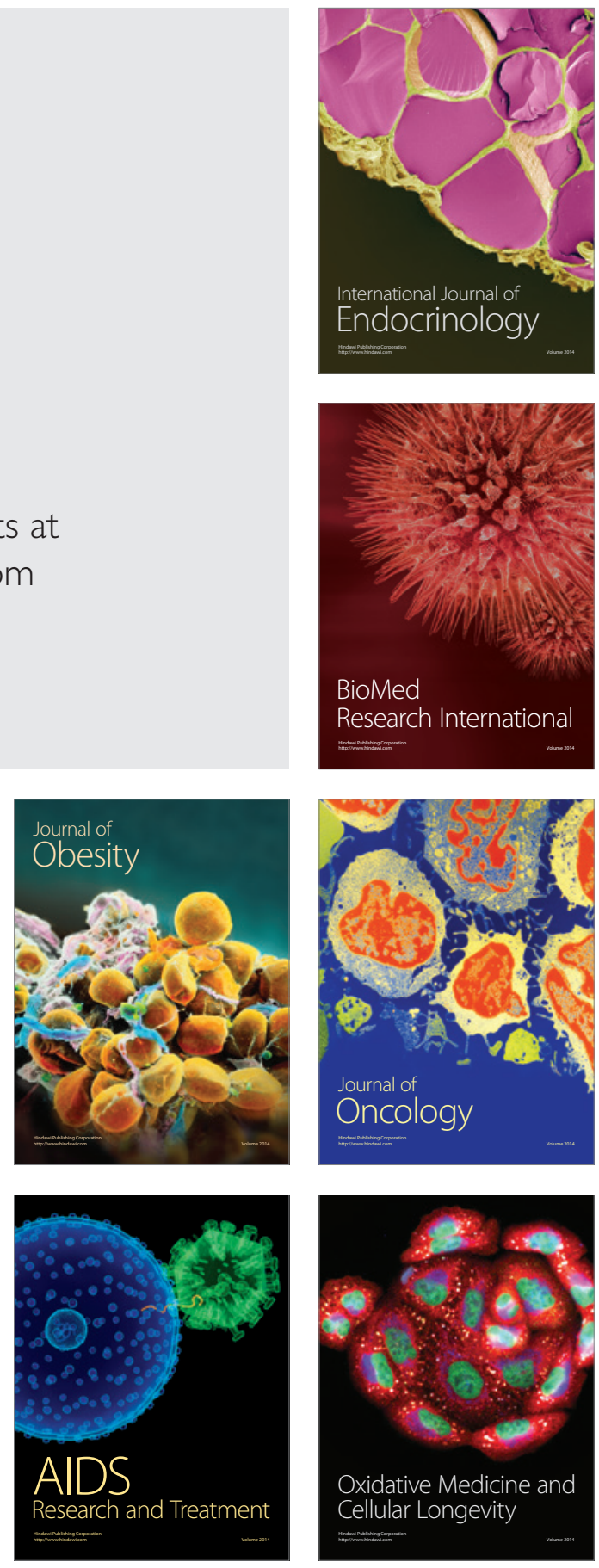